\title{
Synthesis, Thermal Properties, and Gas Permeability of $\operatorname{Poly}(N$-n-alkylmaleimide $)$ s
}

\author{
Akikazu Matsumoto, Yoshitaka OKı, and Takayuki Otsu* \\ Department of Applied Chemistry, Faculty of Engineering, Osaka City University, \\ Sugimoto, Sumiyoshi-ku, Osaka 558, Japan
}

(Received October 25, 1990)

\begin{abstract}
Radical polymerization of $N$ - $n$-alkylmaleimides (nRMI) with various $n$-alkyl groups $(n=4-18)$ was carried out in the presence of $2,2^{\prime}$-azobisisobutyronitrile in benzene at $60^{\circ} \mathrm{C}$. The high molecular weight polymer $\left(\bar{M}_{n}>10^{5}\right)$ was obtained in a high yield from all nRMIs. The resulting poly(nRMI) were soluble in organic solvents such as benzene, chloroform, and THF, and a thin and tough film was obtained from the solution. It was confirmed that poly(nRMI) showed excellent thermal-stability, i.e., they had a high glass transition temperature and decomposition temperatures. The side-chain crystallization was investigated by differential scanning calorimetry. From the measurement of permeation of oxygen and nitrogen through poly(nRMI), it was revealed that these polymers had a high permeability coefficient of $10^{-8}-10^{-9} \mathrm{~cm}^{3}(\mathrm{STP}) \mathrm{cm} \mathrm{cm}^{-2}$ $\mathrm{s}^{-1} \mathrm{~cm} \mathrm{Hg}^{-1}$, which depended intensively on the $N$-substituents.

KEY WORDS $N$ - $n$-Alkylmaleimide / Radical Polymerization / Poly(substituted methylene) / Thermal Stability / Glass Transition Temperature / Side-Chain Crystallization / Differential Scanning Calorimetry / Gas Permeability /
\end{abstract}

It has been reported since 1981 that dialkyl fumarates (DRF) with bulky ester alkyl groups underwent radical high polymerization to give high molecular weight polymers consisting of a poly(substituted methylene) structure. $^{1,2}$ These polymers have a less-flexible structure on account of a defect of a flexible methylene group as a spacer in the main chain, so that they have different properties from flexible ordinary vinyl polymers, i.e., poly(monosubstituted ethylene)s and poly(1,1-disubstituted ethylene)s. ${ }^{2-4}$ For example, poly(DRF)s bearing bulky alkyl groups such as isopropyl or tert-butyl group do not show any glass transition temperature $\left(T_{\mathrm{g}}\right)$ and not melt under their decomposition temperatures. Furthermore, these rigid vinyl polymers have been expected to be applied as a new polymeric material for separation of gases, e.g., an oxygen enrichment membrane. ${ }^{5,6}$ In general, gases such as oxygen and nitrogen permeate in rubbery polymers faster than glassy polymers, ${ }^{7,8}$ but mechanical strength is also an important factor at the same time. Therefore, many efforts have been made for development of a new glassy polymer with high permeability. Poly(1-trimethylsilyl-1-propyne) has been reported to show the highest permeability coefficient, where a large unrelaxed volume in this glassy polymer is important for the high coefficient. ${ }^{9}$

On the other hand, we have reported on radical polymerization of $\mathrm{N}$-alkyl-substituted maleimides and $N$-(alkyl-substituted phenyl)maleimides bearing various alkyl groups, and the excellent thermal-stability of the resulting polymers. ${ }^{10-15}$ Recently, we found that $N$-n-alkylmaleimides (nRMI) polymerized readily as well as other maleimides to give high molecular weight polymer, and that the film of poly(nRMI) obtained showed high permeability for oxygen and nitrogen. ${ }^{16}$ 


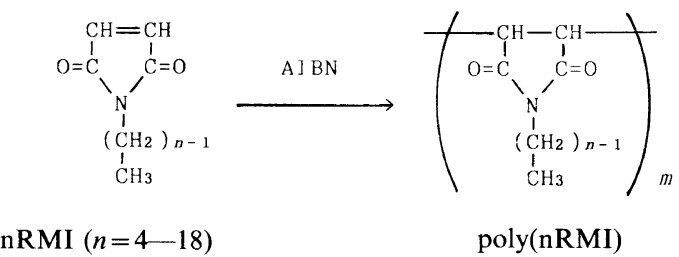

Scheme 1

Therefore, we prepared various $\mathrm{nRMI}$, as shown in Scheme 1, and carried out their radical polymerization. The thermal properties and gas permeability of the resulting poly(nRMI) were investigated.

\section{EXPERIMENTAL}

\section{Materials}

All nRMIs, i.e., $N$ - $n$-butylmaleimide (nBMI), $N$ - $n$-hexylmaleimide (nHMI), $N$-n-octylmaleimide (nOMI), $N$-decylmaleimide (DecMI), $N$-tetradecylmaleimide (TDMI), $N$-hexadecylmaleimide (HDMI), and $N$ - $n$-octadecylmaleimide (ODMI) were prepared from maleic anhydride and a corresponding amine, ${ }^{17,18}$ except for $N$-dodecylmaleimide (DodMI) supplied from Daihachi Chemical Industry Co., Ltd. The nRMIs obtained were purified by distillation or recrystallization from benzene after they were eluted on alumina with benzene. The purity of nRMIs was checked by IR, NMR, and HPLC. The yield and melting point are listed in Table I. Other reagents and solvents were used after ordinary purifications.

\section{Polymerization Procedures}

Polymerization was carried out in the presence of 2,2'-azobisisobutyronitrile (AIBN) in benzene at $60^{\circ} \mathrm{C}$ in a sealed glass tube. The polymerization mixture was poured into large amount of methanol or ethanol to isolate the resulting polymer. The polymer was filtered, washed with methanol or ethanol, and then dried in vacuum. The purification of the polymer was performed by repeating reprecipitation.
Table I. Preparation of nRMI

\begin{tabular}{|c|c|c|c|}
\hline \multirow{2}{*}{ nRMI } & \multirow{2}{*}{$n^{\mathrm{a}}$} & Yield & $\mathrm{mp}$ \\
\hline & & $\%$ & ${ }^{\circ} \mathrm{C}$ \\
\hline nBMI & 4 & 51 & {$[70 / 5 \mathrm{mmHg}]^{\mathrm{b}}$} \\
\hline nHMI & 6 & 15 & 29 \\
\hline nOMI & 8 & 31 & $40(37-37.5)^{\mathrm{c}}$ \\
\hline DecMI & 10 & 20 & $49(46.5-48)^{\mathrm{c}}$ \\
\hline DodMI & 12 & - & $56(54.5-56)^{\mathrm{c}}$ \\
\hline TDMI & 14 & 31 & 64 \\
\hline HDMI & 16 & 30 & 70 \\
\hline ODMI & 18 & 30 & 73 \\
\hline
\end{tabular}

a Number of the carbon in the $\mathrm{N}$-alkyl group.

b Boiling point.

c Lit. (ref 18).

\section{Preparation of Poly(nRMI) Films}

The polymer film, which was used for the measurement of IR, differential scanning calorimetry (DSC), and gas permeability, was prepared by casting of chloroform solution ( $c a$. $10 \mathrm{mg} \mathrm{ml}^{-1}$ ) with slow evaporation of the solvent at room temperature. Subsequently, the film was dried in vacuum at room temperature for a day. The thickness of films was measured with a microgage.

\section{Measurements}

The number- and weight-average molecular weights $\left(\bar{M}_{n}\right.$ and $\left.\bar{M}_{w}\right)$ were determined by gel permeation chromatography (GPC) in THF with standard polystyrenes for calibration.

IR and NMR spectra were recorded on a JASCO A-202 and a Hitachi R-24B or JEOL JNM GX-400 spectrometer, respectively.

Differential scanning calorimetry (DSC) was performed by use of SEIKO DSC-200 or Perkin-Elmer 7 Series thermal analysis systems. The glass transition temperature $\left(T_{\mathrm{g}}\right)$ was determined from thermograms with a heating rate of $20^{\circ} \mathrm{C} \mathrm{min}^{-1}$ after holding at $250^{\circ} \mathrm{C}$ for $5 \mathrm{~min}$ followed by the subsequent quenching. The side-chain crystallization and melting of the crystal were examined by cooling and heating, respectively, in the temperature range of $-150^{\circ} \mathrm{C}$ to $200^{\circ} \mathrm{C}$ with a scanning rate of 
$10^{\circ} \mathrm{Cmin}^{-1}$.

\section{Gas Permeability}

Gas permeation measurements were carried out with ambient air as test gas by means of a Yanaco GTR-10. Partial pressures were calculated from the contents of oxygen $(20.9 \%)$ and nitrogen $(78.1 \%)$ and an atmospheric pressure. The thickness of the films of poly(nRMI) used was $c a .40-250 \mu \mathrm{m}$, and the substantial area for permeation was $15.2 \mathrm{~cm}^{2}$. The measurement was performed at the temperature increased stepwise from $20^{\circ} \mathrm{C}$ up to $50^{\circ} \mathrm{C}$. The amount of the gases (oxygen and nitrogen) which permeated into the downstream pressure across the film was determined by gas chromatography (Yanaco G-1800T) equipped with a column packed with molecular sieve.

\section{RESULTS AND DISCUSSION}

\section{Radical Polymerization}

Table II shows the yield, $\bar{M}_{n}$, and $\bar{M}_{w} / \bar{M}_{n}$ of the polymer obtained from polymerization with AIBN in benzene for $5 \mathrm{~h}$ at $60^{\circ} \mathrm{C}$. All polymerization proceeded easily to give high molecular weight polymers $\left(\bar{M}_{n}>10^{5}\right)$ in high yields $(80-90 \%)$. The time-conversion relation, as depicted in Figure 1, indicates that the polymerization rate increases slightly along with the number of the carbon atoms in the $N$-substituents. It differs from the fact that the introduction of bulky tert-alkyl groups decreased its polymerization rate and $\bar{M}_{n}$ of the polymer as reported previously, ${ }^{14}$ i.e., the polymerization reactivity of $\mathrm{N}$-tert-octylmaleimide (tOMI) was much lower than those of $N$-tert-butylmaleimide (tBMI) or $N$-tert-amylmaleimide (tAMI).

In polymerization of $\mathrm{nRMI}$, it is considered that the higher $\mathrm{N}$-alkyl group does not interfere in propagation, irrespective of the length of the substituents, and tends to decrease relatively the termination rate because of its increasing viscosity of the solution. ${ }^{19}$
Table II. Radical polymerization of $\mathrm{nRMI}^{\mathrm{a}}$

\begin{tabular}{|c|c|c|c|c|c|}
\hline \multirow{2}{*}{ nRMI } & \multirow{2}{*}{$n$} & Yield & $\bar{M}_{n}^{\mathrm{b}}$ & \multirow{2}{*}{$\bar{M}_{w} / \bar{M}_{n}^{\mathrm{b}}$} & $T_{\mathrm{g}}^{\mathrm{c}}$ \\
\hline & & $\%$ & $\times 10^{-5}$ & & ${ }^{\circ} \mathrm{C}$ \\
\hline nBMI & 4 & 80.7 & 1.60 & 2.6 & 185 \\
\hline nHMI & 6 & 80.7 & 1.32 & 2.7 & 148 \\
\hline nOMI & 8 & 85.9 & $1.71(1.73)$ & $2.7(2.0)$ & 138 \\
\hline DecMI & 10 & 87.2 & 1.10 & 2.5 & 131 \\
\hline DodMI & 12 & 88.2 & $1.13(1.62)$ & $2.3(2.0)$ & 117 \\
\hline TDMI & 14 & 86.4 & 1.19 & 2.4 & 109 \\
\hline HDMI & 16 & 89.8 & 1.31 & 2.6 & 103 \\
\hline ODMI & 18 & 88.2 & $(1.70)$ & (2.0) & 97 \\
\hline
\end{tabular}

a Polymerized in benzene at $60^{\circ} \mathrm{C},[\mathrm{nRMI}]=1 \mathrm{moll}^{-1}$, $[\mathrm{AIBN}]=0.05 \mathrm{~mol} \mathrm{l}^{-1}$.

b Determined by GPC. The values in parenthese indicate those for polymers produced in an earlier polymerization stage $(1 \mathrm{~h})$.

c Glass transition temperature, by $\operatorname{DSC}\left(20^{\circ} \mathrm{Cmin}^{-1}\right)$.

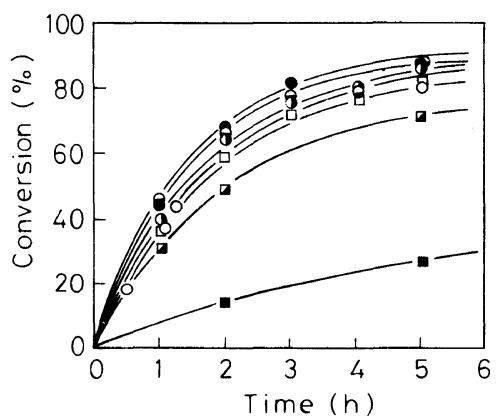

Figure 1. Time-conversion curves for radical polymerization of nRMI: Polymerization conditions, [monomer $]=1 \mathrm{~mol} \mathrm{l}^{-1} ;$ [AIBN] $=5 \times 10^{-3} \mathrm{~mol} \mathrm{l}^{-1}$, in benzene at $60^{\circ} \mathrm{C}$; (O) nBMI; ( ) nOMI; (Ө) DodMI; (○) ODMI; ( $\square)$ tBMI; ( $\square$ ) tAMI; ( $\square$ ) tOMI.

\section{Characterization of the Polymer}

Poly(nRMI) obtained were soluble in many organic solvents, e.g., benzene, chloroform, and THF. Thin films with transparency were obtained by casting of the solutions. They were considerably brittle, but they became more flexible and tough as the number of the carbon atoms in the substituents increased.

It was confirmed that these poly(nRMI) consisted of a poly(substituted methylene) structure, from the ${ }^{1} \mathrm{H}$ and ${ }^{13} \mathrm{C}$ NMR spectra. The IR spectra of the films of poly(nRMI) are shown in Figure 2. The characteristic absorp- 


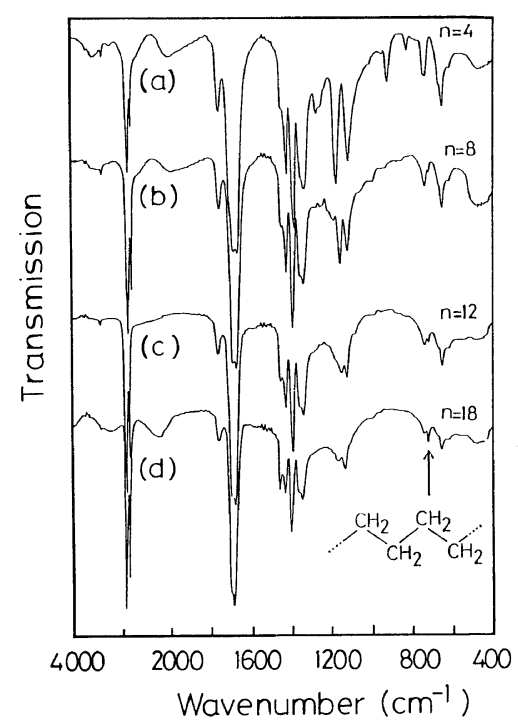

Figure 2. IR spectra of poly(nRMI) films, gained by casting of chloroform solution at room temperature: (a) poly(nBMI); (b) poly(nOMI); (c) poly(DecMI); (d) poly(ODMI).

tions of polymaleimide were observed, and the sharp absorption due to trans zigzag methylenes at $720 \mathrm{~cm}^{-1}$ was additionally detected assignable to the $\mathrm{N}$-alkyl groups. It was found that the intensity of the absorption at 720 $\mathrm{cm}^{-1}$ of poly(ODMI) changed with a thermal treatment, i.e., the peak was broadened immediately when the film was heated at $40^{\circ} \mathrm{C}$. It suggested that the melting of the side chain crystal of poly(ODMI) occurred similarly to other comb-like polymers, as described later.

From the DSC measurements with a heating rate of $20^{\circ} \mathrm{C} \mathrm{min}^{-1}$ as shown in Figure 3, it was revealed that poly(nRMI) had high $T_{\mathrm{g}}$, corresponding to the number of the carbon atoms in the $\mathrm{N}$-alkyl groups, e.g., from $97^{\circ} \mathrm{C}$ for poly(ODMI) $(n=18)$ to $185^{\circ} \mathrm{C}$ for poly(nBMI) $(n=4)$. These $T_{\mathrm{g}}$ values were similar to those reported by Barrales-Rienda et al., ${ }^{20,21}$ being higher than those for other vinyl polymers bearing $n$-alkyl side chains, ${ }^{22}$ e.g., poly $(n$-alkyl acrylate)s, ${ }^{23}$ poly( $n$-alkyl methacrylate $) s,{ }^{24,25}$ poly(di- $n$-alkyl itaconate)s, ${ }^{26}$ and $\operatorname{poly}(N-n$ alkylacrylamide)s. ${ }^{27}$ The stiffness of the poly-

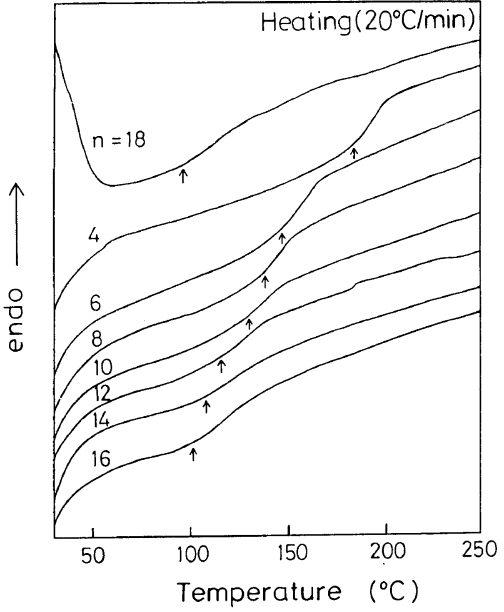

Figure 3. Determination of $T_{\mathrm{g}}$ by DSC with a scanning rate of $20^{\circ} \mathrm{C} \mathrm{min}^{-1}$.

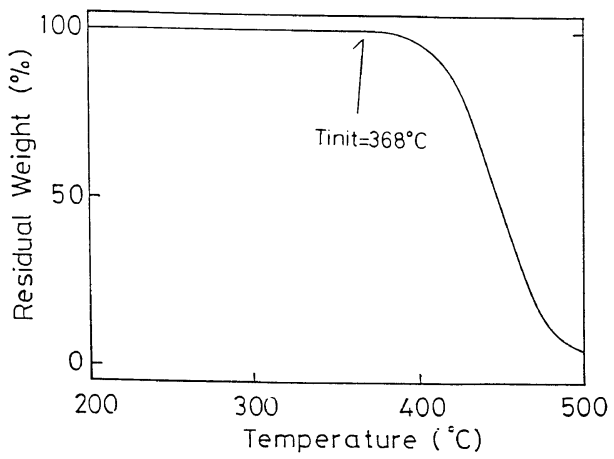

Figure 4. Thermogravimetric analysis of poly(ODMI) in a nitrogen stream with a heating rate of $10^{\circ} \mathrm{C} \mathrm{min}^{-1}$.

mer due to poly(substituted methylene) structure and a rigid imide ring in the main chain is reflected on the high $T_{\mathrm{g}}$ of poly(nRMI).

The thermal decomposition temperatures for poly(nRMI) were also investigated by thermogravimetric analysis in a nitrogen stream with a heating rate of $10^{\circ} \mathrm{Cmin}^{-1}$. The thermogram obtained for poly(ODMI) is shown in Figure 4. The initial and maximum decomposition temperatures were $368^{\circ} \mathrm{C}$ and $445^{\circ} \mathrm{C}$, respectively, indicating that poly(nRMI) have excellent thermal stability as well as other polymaleimides. ${ }^{11}$ 


\section{Side-Chain Crystallization}

The side-chain crystallization of poly(nRMI) was investigated by DSC. When the film of poly(ODMI), which was prepared by casting from a chloroform solution at room temperature, was heated with a heating rate of

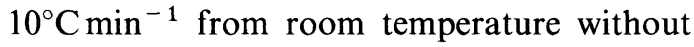
any heating or quenching, an endothermic peak due to melting of the crystalline part in the side chain was observed at $34.6^{\circ} \mathrm{C}$.

Subsequently, poly(ODMI) was once heated up to $200^{\circ} \mathrm{C}$, and then the cooling and heating steps were characterized in the temperature range from $-150^{\circ} \mathrm{C}$ to $200^{\circ} \mathrm{C}$ by $\mathrm{DSC}$. The other poly(nRMI)s were also characterized similarly. The thermograms obtained are shown in Figure 5.

In the cooling steps, exothermic peaks were observed for poly(nRMI) bearing the $N$-alkyl groups with the carbon atoms greater than 10 . Similarly, endothermic peaks were observed in heating. Poly(nRMI) bearing the smaller $N$-alkyl groups $(n<8)$ showed no peaks in the temperature range of $-150^{\circ} \mathrm{C}$ to $200^{\circ} \mathrm{C}$, indicating that their side chains do not crystallize. The melting (fusion) temperature $\left(T_{\mathrm{f}}\right)$ and the crystallization temperature $\left(T_{\mathrm{c}}\right)$ were determined from the top of the endo- and exothermic peaks, respectively, are listed in Table III. These transition temperatures increased with the length of the $\mathrm{N}$-alkyl chain, agreeing with the previous reports by BarralesRienda et al. ${ }^{20,21}$

In the case of poly(ODMI), $T_{\mathrm{f}}$ decreased in the second heating $\left(T_{\mathrm{f}}=19.5^{\circ} \mathrm{C}\right)$, compared with the value $\left(34.6^{\circ} \mathrm{C}\right)$ for the first heating of the as-cast film described above. It was considered that the state of aggregation in the crystalline part had changed. Presumably the crystallization of the alkyl chain from the melting state may be disadvantageous because of the high $T_{\mathrm{g}}$ of the polymer, where the mobility of the side chain was considerably restricted. In casting, on the other hand, the favorable conformation of the both main and side chains of the polymer could be allowed
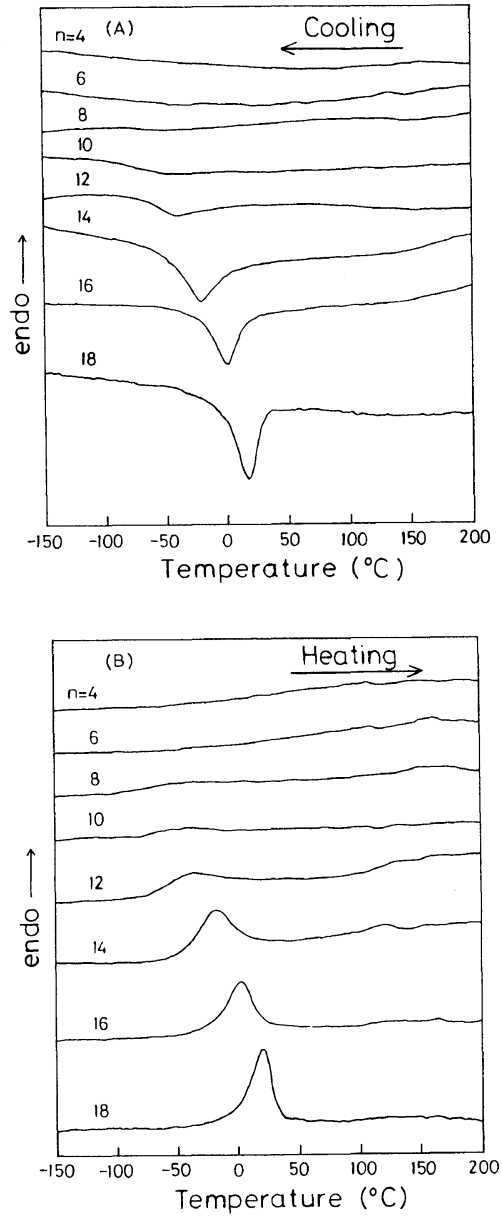

Figure 5. DSC curve of poly(nRMI)s on cooling (A) or heating (B) with a scanning rate of $10^{\circ} \mathrm{C} \mathrm{min}^{-1}$.

because of slow evaporation of the solvent.

The enthalpy change of melting $\left(\Delta H_{\mathrm{f}}\right)$ was determined from the endothermic peaks, and then the entropy change $\left(\Delta S_{\mathrm{f}}\right)$ was calculated therefrom, i.e., $\Delta S_{\mathrm{f}}=\Delta H_{\mathrm{f}} / T_{\mathrm{f}}$. The values obtained are also summarized in Table III.

The values of both $\Delta H_{\mathrm{f}}$, and $\Delta S_{\mathrm{f}}$ increased as the number of $n$ increased. The average number of the methylene units which participated in crystallization $\left(n_{\mathrm{c}}\right)$ was calculated from the relation between $n$ and $\Delta H_{\mathrm{f}}$ as the following equations.

$$
\Delta H_{\mathrm{f}}=\Delta H_{\mathrm{f}, 0}+k n
$$


Table III. Transition temperatures and parameters of the side-chain crystallization for poly(nRMI)

\begin{tabular}{|c|c|c|c|c|c|c|}
\hline \multirow{3}{*}{$n$} & \multirow{2}{*}{$\frac{\text { Cooling }}{T_{\mathrm{c}}^{\mathrm{a}}}$} & \multicolumn{3}{|c|}{ 2nd Heating } & \multirow{3}{*}{$n_{\mathrm{c}}{ }^{\mathrm{d}}$} & \multirow{3}{*}{$n_{\mathrm{a}} \mathrm{e}^{\mathrm{e}}$} \\
\hline & & $\Delta T_{\mathrm{f}}^{\mathrm{b}}$ & $\Delta H_{\mathrm{f}}$ & $\Delta S_{\mathrm{f}}^{\mathrm{c}}$ & & \\
\hline & ${ }^{\circ} \mathrm{C}$ & ${ }^{\circ} \mathrm{C}$ & $\mathrm{kJ} \mathrm{mol}^{-1}$ & $\mathrm{~J} \mathrm{~mol}^{-1} \mathrm{~K}^{-1}$ & & \\
\hline 10 & -49 & -43.0 & 1.34 & 5.82 & 0.9 & 9.1 \\
\hline 12 & -42 & -38.6 & 4.69 & 19.6 & 3.1 & 8.9 \\
\hline 14 & -22 & -17.4 & 7.51 & 29.4 & 5.0 & 9.0 \\
\hline 16 & -1 & 1.5 & 10.4 & 37.9 & 6.9 & 9.1 \\
\hline 18 & 16 & 19.5 & 14.9 & 50.9 & 9.9 & 8.1 \\
\hline
\end{tabular}

a Crystallization temperature of the side chain (by DSC, cooling from $200^{\circ} \mathrm{C}$ to $-150^{\circ} \mathrm{C}$ with a heating rate of $10^{\circ} \mathrm{C} \mathrm{min}^{-1}$ ).

b Melting temperature of the side-chain crystal (by DSC, heating from $-150^{\circ} \mathrm{C}$ to $200^{\circ} \mathrm{C}$ ith a heating rate of $10^{\circ} \mathrm{C} \mathrm{min}^{-1}$ ).

c Calculated from $T_{\mathrm{f}}$ and $\Delta H_{\mathrm{f}}$.

d The number of the carbon atoms of the side chain involved in the crystal; from eq 2 .

e The number of the carbon atoms of the side chain involved in the amorphous part; from eq 3 .

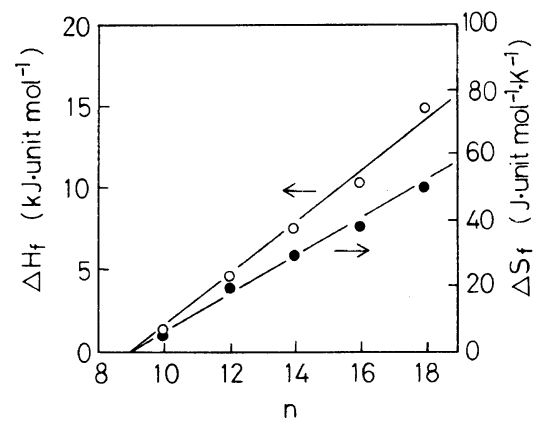

Figure 6. The relation between the enthalpy $\left(\Delta H_{\mathrm{f}}\right)$ or entropy change $\left(\Delta S_{\mathrm{f}}\right)$ and the number of the carbon atoms (n) of the side-chain for poly(nRMI)s.

$$
n_{\mathrm{c}}=\Delta H_{\mathrm{f}} / k
$$

Where $\Delta H_{\mathrm{f}, 0}$ is the contribution of the end groups of the side chains to the enthalpy of fusion. From the slope of the line in Figure 6, the enthalpy of melting per a methylene unit (k) was calculated to be $1.5 \mathrm{~kJ}$, which was lower than values for the methylene in the side-chain crystal of other polymers ${ }^{22,28-31}$ or the crystal of $n$-alkanes with hexagonal packing, ${ }^{32,33}$ and also the value for the same polymer by BarralesRienda et al. $(3.21 \mathrm{~kJ}){ }^{21}$ It suggests that the side-chain crystal in poly(nRMI) is far from a complete crystal under our DSC conditions, i.e., a scanning rate of $10^{\circ} \mathrm{Cmin}^{-1}$. It may be attributed to the rigidity of the main chain of poly(nRMI), which obstructs reorientation of the alkyl side chains.

The $n_{\mathrm{c}}$ determined are shown in Table III, in which the number of methylene units in amorphous $\left(n_{\mathrm{a}}\right)$ (eq 3$)$ is also included.

$$
n_{\mathrm{a}}=n-n_{\mathrm{c}}
$$

The value of $n_{\mathrm{a}}$ indicates that the 9 carbons in the $N$-alkyl groups can not participate in crystallization. Similar values have been also reported for the many kind of polymers with alkyl sidechain capable of crystallization. ${ }^{22,28-35}$

\section{Gas Permeability}

The permeability of oxygen and nitrogen through poly(nRMI) was investigated with stepwise heating in the temperature range of $20^{\circ} \mathrm{C}$ to $50^{\circ} \mathrm{C}$. The results are shown in Figures 7-9. The permeability coefficients $\left(P_{\mathrm{O}_{2}}\right.$ and $\left.P_{\mathrm{N}_{2}}\right)$ and the selectivity $\left(P_{\mathrm{O}_{2}} / P_{\mathrm{N}_{2}}\right)$ at $40^{\circ} \mathrm{C}$ are summarized in Table IV. The permeability coefficients obtained were in the range of $10^{-8}$ to $10^{-9} \mathrm{~cm}^{3}$ (STP) $\mathrm{cm} \mathrm{cm}^{-2} \mathrm{~s}^{-1} \mathrm{~cm} \mathrm{Hg}^{-1}$, be much higher than any other glassy vinyl polymers, and similar to those for rubbery polymers. ${ }^{7,8}$ The selectivity for the permeation of oxygen to nitrogen was $2-2.5$. The overall 

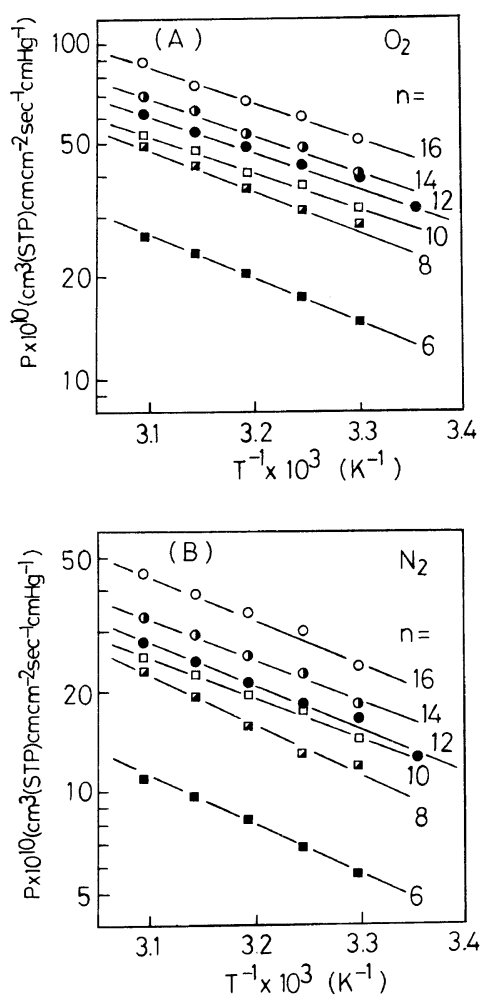

Figure 7. Temperature dependence of gas permeability coefficients for poly(nRMI): (A) oxygen; (B) nitrogen. (O) poly(HDMI); (O) poly(TDMI); (O) poly(DodMI); ( $\square$ ) poly(DecMI); ( $\boldsymbol{\square})$ poly(nOMI); ( $)$ poly(nHMI).

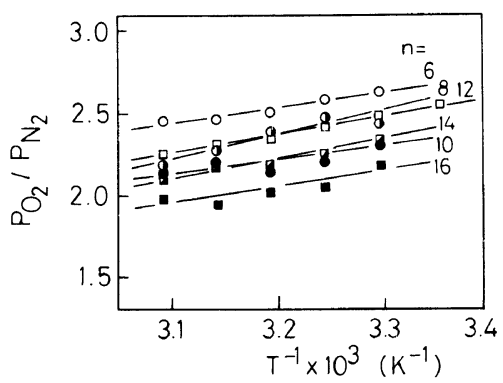

Figure 8. Permselectivity of oxygen to nitrogen for poly(nRMI)s.

activation energies for permeation, estimated from the relations between $\log P$ and the reciprocal of temperature in Figure 7 , were $21-24 \mathrm{~kJ} \mathrm{~mol}^{-1}$ for oxygen and $25-27 \mathrm{~kJ}$ $\mathrm{mol}^{-1}$ for nitrogen.

It was revealed that the permeability

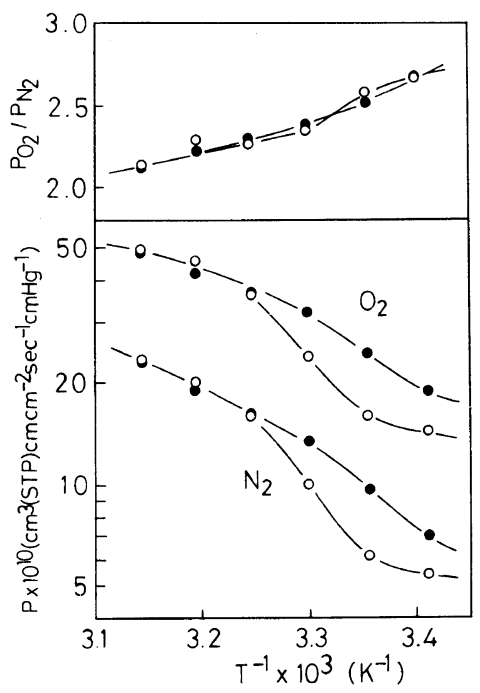

Figure 9. Temperature dependence of gas permeability coefficients and selectivity for poly(ODMI): (O) at 1st heating; (O) 2nd heating.

Table IV. Gas permeability coefficients $\left(P_{\mathrm{O}_{2}}, P_{\mathrm{N}_{2}}\right)$ and selectivity $\left(P_{\mathrm{O}_{2}} / P_{\mathrm{N}_{2}}\right)$ for poly(nRMI) at $40^{\circ} \mathrm{C}$

\begin{tabular}{lrrrr}
\hline & \multicolumn{4}{c}{$P^{\mathrm{a}}$} \\
\cline { 3 - 5 } & $n$ & $P_{\mathrm{O}_{2}} / P_{\mathrm{N}_{2}}$ \\
\cline { 3 - 5 } & & $\mathrm{O}_{2}$ & $\mathrm{~N}_{2}$ \\
\hline Poly(nHMI) & 6 & 20.6 & 8.25 & 2.50 \\
Poly(nOMI) & 8 & 37.3 & 15.6 & 2.39 \\
Poly(DecMI) & 10 & 41.5 & 19.5 & 2.13 \\
Poly(DodMI) & 12 & 49.2 & 20.9 & 2.35 \\
Poly(TDMI) & 14 & 53.4 & 25.1 & 2.13 \\
Poly(HDMI) & 16 & 67.4 & 34.1 & 1.99 \\
Poly(ODMI) & 18 & 46.3 & 20.3 & 2.29 \\
\hline a $P$ in $10^{-10} \mathrm{~cm}^{3}(\mathrm{STP}) \mathrm{cm} \mathrm{cm}^{-2} \mathrm{~s}^{-1} \mathrm{~cm} \mathrm{Hg}^{-1}$.
\end{tabular}

increased according to the carbon number in the side chain except for poly(ODMI), suggesting that the contribution of the side alkyl chain may be important for the permeability. On the other hand, poly(ODMI) gave exceptionally the curves different from the linear relations for other polymers for both oxygen and nitrogen, as shown in Figure 9. When the first measurement was carried out with stepwise heating, the permeability increased non-continuously at around $30^{\circ} \mathrm{C}$. Furthermore, the permeability increased in the 


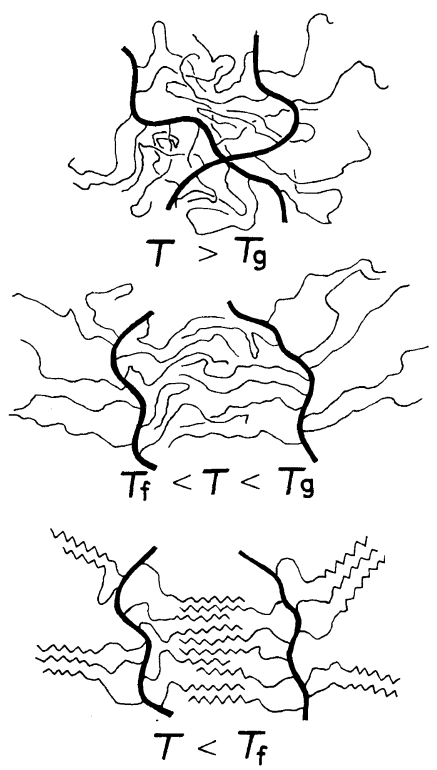

Figure 10. Change in the supposed structure of the alkyl side-chain of poly(nRMI).

second heating than those for the first heating. This was attributed to the difference in the state of aggregation of the side chains, as mentioned on the DSC study above. It was considered that the permeability coefficient at $40^{\circ} \mathrm{C}$ for poly(ODMI) was smaller than those poly(TDMI) or poly(HDMI) on account of the effect of the side-chain crystallization, since the transition temperature was close to the measurement temperature. From these results, the following model was proposed for the permeation through poly(nRMI).

The supposed structure of the poly(nRMI) with long alkyl side-chain is depicted in Figure 10. The side chain of poly(nRMI) is considered to have some ordered structure at the temperature between $T_{\mathrm{g}}$ and $T_{\mathrm{f}}$ because of the immobility of the semiflexible main chain, and a part of the methylene units can crystallize under its $T_{\mathrm{f}}$. The high gas permeability is ascribed to the alkyl side-chain with highly active segmental motion. The methylenes in the alkyl side-chain apart from the main chain have high mobility, so that a simple solubility and diffusion mechanism might be dominant, similarly to permeation through rubbery polymers. In fact, we have recently found that the permeability coefficient of poly(nRMI) depended hardly on the pressure of the up-stream side, suggesting that the permeation is apparently not governed by a dual-mode transport mechanism, ${ }^{36}$ which is used widely for the interpretation of permeation behavior through many glassy polymers, although poly(nRMI)s have high $T_{\mathrm{g}}$ because of the rigidity of the main chain. However, much free volume in the neighborhood of the semiflexible main chain of poly(nRMI) might not be negligible. Further discussions on the mechanism of permeation through poly(nRMI) will be reported elsewhere.

\section{CONCLUSION}

Radical polymerization of nRMI with various $N$-alkyl groups $(n=4-18)$ proceeded readily to give a high molecular weight polymer in a high yield. The poly(nRMI)s were soluble in many organic solvents such as benzene and chloroform, and a thin film was obtained by casting of the solution. It was revealed that poly(nRMI) had high $T_{\mathrm{g}}$ respective to the length of the $N$-alkyl chain $\left(97-185^{\circ} \mathrm{C}\right)$, and high decomposition temperatures, e.g., onset of weight loss was $368^{\circ} \mathrm{C}$ for poly(ODMI) in thermogravimetric analysis in nitrogen. The side-chain crystallization was observed for poly(nRMI) bearing long alkyl chain as $N$ substituents $(n>10)$ by DSC. From the melting temperature and enthalpy changes of the sidechain crystal of the polymers, the entropy changes and the number of the methylene unit involved in the crystals were calculated. From the results for measurements of permeability of oxygen and nitrogen through poly(nRMI), it was found that the permeability coefficients were more than $10^{-9} \mathrm{~cm}^{3}(\mathrm{STP}) \mathrm{cm} \mathrm{cm}^{-2} \mathrm{~s}^{-1}$ $\mathrm{cm} \mathrm{Hg}^{-1}$, and depended on the length of the side chain. Moreover, it was also verified that the side-chain crystallization decreased markedly the permeability. 
Acknowledgments. The authors are indebted to Polymer Research Laboratory, Tosoh Co. for DSC measurement, and to Daihachi Chemical Industry Co., Ltd. for supply of $N$-dodecylmaleimide. A part of this work was supported by a Grant-in-Aid for Scientific Research (No. 01430019) and Encouragement of Young Scientist (No. 02750651) from the Ministry of Education, Science and Culture, Japan.

\section{REFERENCES}

1. T. Otsu, O. Ito, N. Toyoda, and S. Mori, Makromol. Chem., Rapid Commun., 2, 725 (1981).

2. T. Otsu, T. Yasuhara, and A. Matsumoto, $J$. Macromol. Sci-Chem., A25, 537 (1988), and references therein.

3. A. Matsumoto, T. Tarui, and T. Otsu, Macromolecules, 23, 5102 (1990).

4. M. Yoshioka, A. Matsumoto, T. Otsu, and I. Ando, Polymer, in press.

5. H. Ikebe, Y. Murata, and T. Otsu, Polym. Prepr., Jpn., 34, 420 (1985).

6. S. B. Choi, A. Takahara, N. Amaya, Y. Murata, and T. Kajiyama, Polym. J., 21, 433 (1989).

7. W. J. Koros, G. K. Fleming, S. M. Jordan, T. H. Kim, and H. H. Hoehn, Prog. Polym. Sci., 13, 339 (1988).

8. H. Yasuda and V. Stannett, "Polymer Handbook," 2nd ed, J. Brandrup and E. H. Immergut, Ed., Wiley, New York, N.Y., 1975, pp III-229.

9. T. Masuda, E. Isobe, T. Higashimura, and K. Takada, J. Am. Chem. Soc., 105, 7473 (1983); K. Takada, H. Matsuya, T. Masuda, and T. Higashimura, J. Appl. Polym. Sci., 30, 1605 (1985); T. Masuda, Y. Iguchi, B. Z. Tang, and T. Higashimura, Polymer, 29, 2041 (1988).

10. T. Otsu, A. Tatsumi, and A. Matsumoto, J. Polym. Sci., Polym. Lett. Ed., 24, 113 (1986).

11. T. Otsu, A. Matsumoto, T. Kubota, and S. Mori, Polym. Bull., 23, 43 (1990).

12. A. Matsumoto, T. Kubota, and T. Otsu, Macromolecules, 23, 4508 (1990).

13. A. Matsumoto, T. Kubota, and T. Otsu, Polym. Bull., 24, 459 (1990).

14. T. Otsu, A. Matsumoto, and A. Tatsumi, Polym. Bull., 24, 467 (1990).
15. T. Otsu, A. Matsumoto, and T. Kubota, Br. Polym. $J$, in press.

16. A. Matsumoto, Y. Oki, and T. Otsu, Markromol. Chem. Rapid Commun., 11, 507 (1990).

17. N. B. Mehta, A. R. Phillips, F. F. Lui, and R. E. Brooks, J. Org. Chem., 25, 1012 (1960).

18. L. E. Colleman, Jr., J. F. Bork, and H. Donn, Jr., J. Org. Chem., 24, 185 (1959).

19. T. Otsu, O. Ito, and M. Imoto, J. Polym. Sci., A2, 2901 (1964).

20. J. M. Barrales-Rienda, J. G. Ramos, and M. S. Chaves, Br. Polym. J., 9, 6 (1977).

21. J. M. Barrales-Rienda, F. Fernandez-Martin, C. R. Garcia, and M. S. Chaves, Makromol. Chem., 184, 2643 (1983).

22. N. A. Plate and V. P. Shibaev, Macromol. Rev., 8, 117 (1974).

23. C. E. Rehberg and C. H. Fisher, J. Am. Chem. Soc., 66, 1203 (1944).

24. S. A. Greenberg and T. Alfrey, J. Am. Chem. Soc., 76, 6280 (1954).

25. S. S. Rogers and L. Manderkern, J. Chem. Phys., 61, 985 (1957).

26. J. M. G. Cowie, S. A. E. Henshall, I. J. McEwen, and J. Velichovic, Polymer, 18, $6 \mathrm{i} 2$ (1977).

27. E. F. Jordan, Jr., G. R. Riser, B. Artymyshyn, W. E. Parker, J. W. Pensabene, and A. N. Wrigley, J. Appl. Polym. Sci., 13, 1777 (1969).

28. E. F. Jordan, Jr., D. W. Feldeisen, A. N. Wrigley, $J$. Polym. Sci., A-1, 9, 1835 (1971).

29. J. Watanabe, H. Ono, I. Uematsu, and A. Abe, Macromolecules, 18, 2141 (1985).

30. J. I. Gonzalez de la Campa, J. M. Barrales-Rienda, and J. G. Ramos, J. Polym. Sci., Polym. Phys. Ed., 18, 2197 (1980).

31. J. M. Barrales-Rienda and M. Mazon-Arechederra, Macromolecules, 20, 1637 (1987).

32. E. F. Jordan, Jr., J. Polym. Sci., Polym. Chem. Ed., 10, 3347 (1972).

33. C. M. L. Atkinson and M. J. Richardson, Trans. Faraday Soc., 65, 1749 (1969).

34. T. Hirabayashi and K. Yokota, Polym. J., 19, 1115 (1987); T. Hirabayashi, T. Kikuta, K. Kobayashi, and K. Yokota, ibid., 20, 693 (1988).

35. J.-K. Lin, Y. Yuki, H. Kunisada, M. Kondo, and S. Kondo, Polym. J., 22, 47 (1990); H. Kunisada, Y. Yuki, S. Kondo, J. Miyatake, and C. Maeda, ibid., 22, 559 (1990).

36. D. R. Paul and W. J. Koros, J. Polym. Sci., Polym. Phys. Ed., 14, 675 (1976). 